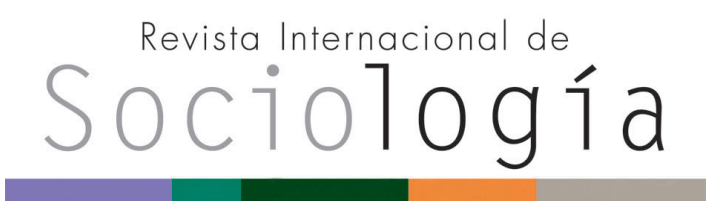

Revista Internacional de Sociología RIS

vol. 74 (3), e036, julio-septiembre, 2016, ISSN-L:0034-9712

doi: http://dx.doi.org/10.3989/ris.2016.74.3.036

\section{FORMAS MODERNAS DE RESACRALIZACIÓN EN DISPUTA La nación y la persona}

\author{
JosetXo BERIAIN \\ Universidad Pública de Navarra, España. \\ josetxo@unavarra.es
}

Cómo citar este artículo / Citation: Beriain, J. 2016. "Formas modernas de resacralización en disputa. La nación y la persona", Revista Internacional de Sociología 74 (3): e036. doi: http://dx.doi.org/10.3989/ris.2016.74.3.036

\section{THE STRUGGLE AMONG MODERN FORMS OF RESACRALIZATION The nation and the human person}

Copyright: (C) 2016 CSIC. Este es un artículo de acceso abierto distribuido bajo los términos de la licencia Creative Commons Attribution (CC BY) España 3.0.
Recibido: 17/09/2014. Aceptado: 01/07/2015. Publicación online: 20/07/16

\section{Abstract}

This paper takes into account -against a canonical and teleological approach of the general theory of secularization which is born within the western Christianity and according to it religion is a vestige of the past in an stage of modernity fully developed- the analysis of modern resacralization phenomena such as the nation and the person. On the one hand, three case studies as the French Revolution, the American Civil religion and the ritual mobilizations that follow the attack against the WTC in the 9/11 show us the empirical support to explain the emergence of multiple postaxial sacred forms. On the other hand, the modern resacralization of an other secular sphere, the human person, set off an other cultural dynamics. Finally, there have been analyzed two cases of modern societies where those modern resacralizations are projected as symbolic cleavages.

\section{KEYWORDS}

Narrative; Profane; Sacralization; Sacred; Value Struggle. 


\section{INTRODUCCIÓN}

El objetivo central de este artículo es acometer la tarea de esbozar una "genealogía afirmativa"1 de los fenómenos de resacralización moderna que se producen en dos ámbitos seculares, como son la nación y la persona. Para abordar este objetivo analizo la génesis social del hecho religioso, diferenciando este planteamiento: a) de las teorías animistas y naturalistas de la religión -asumiendo los supuestos durkheimianos, según los cuales el hecho religioso no hace referencia a seres sobrenaturales o misteriosos- b) de las formulaciones ontologistas, representadas por Rudolph Otto, según las cuales el mundo trascendente funge como realidad ontológicamente suprema y dada, y c) de la teoría general de la secularización, que tiene su origen en el cristianismo europeo-occidental, y que ha proyectado una "conjetura sociológica" que funge de "régimen de conocimiento" con connotaciones no solo descriptivas sino prescriptivas y que podíamos formular de la siguiente manera: "cuanto más moderna, más secular es una sociedad, y cuanto más secular, menos religiosa”. A modo de hipótesis, en la "genealogía afirmativa" que propongo, el hecho religioso no es una reliquia o un atavismo de un estadio superado sino que las transformaciones del hecho religioso están asociadas a la génesis histórico-social de pares de categorías -sagrado/ profano, trascendente/inmanente, secular/religioso- ${ }^{2}$ que interactúan en tensión dinámica y conforman situaciones nuevas en las que, siguiendo la metáfora de la física, la energía no se pierde; la "creatividad social", la "capacidad de trascendencia", produce una nueva síntesis de realidades instituidas e instituyentes, sin hacer tabula rasa, sin restar, sino sumando de forma creativa. Aquí radica el dinamismo del hecho religioso, no tanto en su pasado como en su pasado futuro en su hacerse histórico-social. En el momento actual se da "por una parte, una coexistencia entre diferentes religiones y, por otra parte, una coexistencia entre discursos religiosos y discursos seculares" (P. L. Berger 2014: 9). Con la hipótesis de una "genealogía afirmativa" del hecho religioso de fondo, analizo tres casos distintos como son la Revolución Francesa, la religión civil americana y la sacralidad emergente tras el ataque al WTC, el 9/11/2001, en donde se manifiestan procesos de sacralización de una realidad secular como es la nación. Pero, se ha producido una sacralización de otra realidad secular, casi de forma paralela, la persona humana, que se objetivó en las diversas declaraciones de los derechos humanos (1789 y 1948). En el último apartado, analizo cómo la emergencia de estas "nuevas formas sagradas múltiples postaxiales" no representa una estructura pluralista, funcional y armónica, tal como la han interpretado Parsons, Shils y Warner, desde la perspectiva funcionalista, sino que supone la "tensión dinámica y en conflicto" entre tales múltiples formas sagradas, y ejemplifico este argumento a través de dos estudios de caso tomados de la historia reciente de Irlanda y del País Vasco. En estos ejemplos se pone de manifiesto el "choque" de las narrativas que sacralizan la nación con las narrativas que sacralizan a la persona humana.

\section{LA TRASCENDENCIA INMANENTE COMO CARACTERÍSTICA PRIMORDIAL DEL HECHO RELIGIOSO}

Para comenzar a desvelar las formas sagradas modernas, conviene desvelar previamente el "núcleo central común que caracteriza la génesis social" que adoptan las distintas formas del hecho religioso.

En orden a constituirse como sujeto viable, uno debe "trascenderse" a sí mismo, debe experimentar -en una multitud de formas y contextos- cómo algo se apodera de uno, cómo se quiebra "lo dado por supuesto", lo habitual, lo rutinario, alumbrando una situación de ruptura, de apertura de algo más allá de uno mismo, que puede ser (o no ser) interpretado religiosamente. Este estado se puede alcanzar en el mundo de la fantasía, en los sueños, en el éxtasis, en la actitud de reflexión teórica profunda, y en el ritual religioso. La trascendencia es un a priori antroposocial cuya presencia en la vida humana no es algo exclusivo de la narrativa religiosa, aunque es en esta última donde más se ha desarrollado. Según Simmel (2000: 89, 299, 304-305, 309-312), la referencia a los límites, pone de manifiesto, de algún modo, la posibilidad de rebasarlos, que de hecho sucede más tarde o más temprano. Es un hecho que "trascendemos" el mundo de la realidad sensible, el mundo de la experiencia cotidiana, en el pensamiento, en la fantasía, en los sueños, en la religión, hasta considerar sus límites "desde fuera".

El límite, en cuanto tal, participa del aquende y del allende, de modo que el acto unificado de la vida incluye ambos momentos, el del ser limitado y el de la "trascendencia" del límite. "Es esencial para el hombre, en lo más profundo, el hecho de que él mismo se ponga una frontera, pero con libertad, esto es, de modo que también pueda superar nuevamente esta frontera, situarse más allá de ella" (Simmel 1986: 31), porque "somos a cada instante aquellos que separan lo ligado o ligan lo separado" (ibídem, p. 29). Somos seres fronterizos sin ninguna frontera (ibídem, p. 34).

En lenguaje bergsoniano podemos decir que la realidad no se reduce a lo actualmente existente sino que abarca el conjunto de posibilidades co-dadas en el presente, que precisan poder-ser realizadas trascendiendo, transgrediendo, cruzando determinados límites. Estos solo son reconocidos, solo podemos hablar de los "límites de la experiencia" en la medida en que se da una cierta "experiencia de los límites" de la realidad (Schutz y Luckmann 1984: 142-147); solo cuando "cruzamos" un límite y nos situamos al otro lado podemos hablar de una experiencia del límite, teniendo siempre presente que existe un límite final de la experiencia, la muerte, cuyo conocimiento 
es indudable, puesto que sabemos que moriremos, pero, sin embargo, no es indudable aquello que está más allá de la muerte, como bien apunta Shakespeare en su Hamlet.

No habitamos "un" mundo clausurado, sino que vivimos en medio de un conjunto de "realidades múltiples en plural" conectadas de alguna manera, como observó Alfred Schutz (1974: 215 y ss.) a partir de ideas previas de William James. Estas realidades configuran ámbitos finitos de sentido, sobre los que proyectamos un determinado acento de realidad. La realidad del mundo de la vida cotidiana (profana en los términos de Durkheim) se nos aparece como la realidad natural, y no estamos dispuestos a abandonar nuestra actitud hacia ella sin haber experimentado una "conmoción" específica que nos obligue a trascender los límites de ese ámbito "finito" de sentido y trasladar a otro el acento de realidad. La transición del mundo cotidiano ordinario al mundo religioso extraordinario solo puede ser efectuada mediante un "salto", como lo Ilama Kierkegaard, una "conmoción”, una modificación radical en la tensión de nuestra conciencia. Determinados tipos ideales -el mago-mistagogo, el profeta, el sacerdote, el líder carismático-ayudan a realizar este tránsito, como ha demostrado Max Weber en su sociología de la religión.

Durkheim sitúa este "salto" en la práctica ritual como ese "acontecimiento apropiador" que produce un tipo de realidad diferente. La "efervescencia colectiva" ${ }^{3}$ el "éxtasis colectivo", la "energía emocional", es esa condición de posibilidad a través de la cual la gente experimenta una realidad diferente y más profunda. Así afirma que: "una vez alcanzado tal estado de exaltación, el hombre pierde la conciencia de sí mismo. Sintiéndose dominado, arrastrado, por una especie de fuerza exterior que le hace pensar y actuar de modo distinto a como lo hace normalmente, tiene naturalmente la impresión de haber dejado de ser él mismo. Le parece que se ha convertido en un "nuevo ser": las galas con que viste, las especies de máscaras con las que se cubre la cara, son representaciones materiales de esta transformación,...Y como, al mismo tiempo, todos sus compañeros se sienten trasfigurados de la misma manera y exteriorizan su sentimiento en sus gritos, gestos y actitudes, todo se desarrolla como si realmente fuera transportado a un mundo especial, completamente diferente de aquel en que vive de ordinario, a un espacio poblado por completo de fuerzas excepcionalmente intensas, que le invaden y metamorfosean"4. Durkheim nos habla de un nuevo modo de ser y de un mundo especial. "Solo a través del acontecimiento apropiador del ritual emerge lo sagrado como algo diferenciado de lo profano". De esta guisa se crean dos mundos, o más precisamente, un mundo se diferencia y genera dos ámbitos de realidad distintos. De hecho, la razón para la rea- lización performativa de tales prácticas rituales es mantener el límite entre lo sagrado y lo profano, no tanto una creencia en seres sobrenaturales o en dioses misteriosos, como pretenden las concepciones convencionales de la religión. Lo sagrado no existe como una idea o creencia previa, solo a través del ritual viene a la existencia. La existencia de lo sagrado precede a la esencia en lo sagrado.

Frente a la posición ontologista neoplatónica de Rudolph Otto (1985: 38 y ss.) que considera lo sagrado (Das Heilige) como un mysterium tremendum fascinans dado, pre-existente, defendemos la génesis social de un contramundo a partir de la experiencia trasgresora de trascendencia inmanente que tiene lugar en el ritual. El hecho religioso se encarna en una "comunidad de culto" antes que representarse en una "comunidad de interpretación y de creencia".

En las sociedades modernas, las antiguas formas sagradas no han muerto -a diferencia de lo que Durkheim pensó- y han nacido otras formas sagradas específicamente modernas que compiten con aquellas, entre sí y con los discursos seculares, en una lucha sin fin. Hablar de lo sagrado en las sociedades modernas significa mirar a las situaciones históricas contingentes (Th. A. Tweed 2006: 54-79) que crean formas específicas de trascendencia. Aunque el mundo inmanente secular, ayudado por el discurso secularista auspiciado por el Estado moderno, ha creado reglas de juego vinculantes más allá de toda creencia y práctica religiosas, sin embargo, no ha creado una realidad postdualista y postreligiosa sino que han surgido múltiples formas sagradas en tensión dinámica entre sí y con las formas seculares.

Las sociedades modernas no conforman una colectividad homogénea en sus creencias dentro de la cual sus miembros mantienen una única referencia simbólica, a la manera del modelo de integración social simple que Durkheim describe en las sociedades tribales, sino que existe un "elenco múltiple de formas sagradas y seculares" (G. Lynch 2012: 135), debido a que "la creencia en Dios ya no es algo axiomático, (de que) hay alternativas" (Ch. Taylor 2007: 3). Hemos pasado de una sociedad donde era virtualmente imposible no creer en Dios, o, al menos, no contar con el axioma de la creencia en Dios como eje cardinal del sentido común (porque estaba socialmente prescrito creer y proscrito el no creer, en un contexto masivamente creyente), a una donde la fe, incluso para el más radical de los creyentes, es una posibilidad (loc. cit.) humana entre otras. Charles Taylor, recogiendo la fórmula de Hugo Grocio, afirma que este hecho sociológico crucial producirá un nuevo patrón de significado según el cual actuamos dentro de un "marco inmanente secular" bajo la premisa: etsi Deus non daretur (Ch. Taylor 1998: 34 y 36) ("como si Dios no existiera"); incluso si os no existiera, los principios que emanan de tal "mundo inmanente secular" son vinculantes. Se podría decir que en el seno del sae- 
culum emerge una nueva distinción directriz, la que diferencia entre un "nosotros" (creyentes religiosos guiados por su fe, sea la que fuere) y el "ellos" (los sujetos postreligiosos que se rigen según pretensiones racionales de validez). Esto de ninguna manera significa que el ser humano haya dejado de ser religioso, sino, más bien, que determinadas cosas que pensamos o hacemos no tienen naturaleza religiosa ni precisan ser explicadas en términos religiosos; o, en otras palabras, que se establece una "geometría variable de opciones entre las esferas sagradas y las seculares. La diferenciación no solo existe dentro del propio hecho religioso -presente en la multitud de nuevas formas sagradas emergentes- sino que afecta a la diferenciación entre los hechos religiosos y los hechos seculares igualmente diferenciados".

\section{TRES HITOS MODERNOS DE RESACRALIZACIÓN DE LA REALIDAD SECULAR DE LA NACIÓN}

\section{La Revolución Francesa}

Aunque podíamos presentar como soporte empírico de nuestra hipótesis de partida todo un conjunto de estudios de caso -que puede englobar desde la Revolución Francesa, pasando por los ritos que agrupan la religión civil americana y las movilizaciones rituales que siguen al ataque al WTC el 9/11, incluyendo también en nuestro análisis la efervescencia colectiva que lleva a la unificación postotalitaria de Europa en la posguerra y la ceremonia de coronación de la reina Isabel II- voy a circunscribir mi análisis al estudio de los tres primeros casos, a que ofrecen suficientes elementos para ejemplificar razonablemente la emergencia de un proceso de resacralización moderna de la nación. Comenzando con nuestra particular "genealogía afirmativa" de las expresiones de resacralizaciones modernas que afectan a la nación, me voy a detener en el caso de la Revolución Francesa (Lynn Hunt 1988: 25-44; Edward Tiryakian 1988: 44-66). El abate Sieyés, en su celebrado, Qu'est ce que le TiersEtat, publicado en 1789, afirmó que la nación francesa era "un cuerpo de asociados que viven bajo leyes comunes y representados por la misma asamblea legislativa [...] Fue algo anterior, preexistente a todos los fenómenos e instituciones sociales [...] La imagen de la Patrie es la única a la que rendir culto" (1970: 1011). Según este fragmento, podemos observar cómo la comunidad de salvación -propia de las religiones universales- se transforma en una comunidad de culto, de prácticas, en una comunidad nacional imaginada, en los términos de Benedikt Anderson, en la que el nuevo objeto de culto es la nación, o mejor dicho, "el pueblo de la nación". La "efervescencia colectiva" que origina la trascendencia del mundo ordinario crea un plus extra-ordinario en medio de la intensificación de la vida social creada por la Revolución Francesa, marcando el origen de la nación como la expresión protomoderna de la identidad colectiva.
A juicio de Durkheim, no habría una diferencia sustancial en cuanto a la forma ritual entre la reunión de los cristianos que celebran los principales hitos de la vida de Cristo, los judíos que recuerdan el Éxodo de Egipto, una reunión de ciudadanos que conmemoran la promulgación de una nueva moral o un nuevo sistema legal o un acontecimiento significativo de la vida nacional. De la Revolución Francesa emergió una voluntad constituyente representada en una nueva fe cuyos principios están contenidos en la Declaración de los Derechos del Hombre y del Ciudadano (A. Mathiez 1904; 2012)5 . Lo "real-regio" se convirtió en lo "nacional". La referencia durkheimiana es reveladora a este respecto: "Esta capacidad de la sociedad para erigirse en un dios o para crear dioses no fue en ningún momento más perceptible que durante los primeros años de la Revolución Francesa. En aquel momento, en efecto, bajo la influencia del entusiasmo general, cosas puramente laicas fueron transformadas por parte de la opinión pública en cosas sagradas, así la Patria, la Libertad, la Razón. Hubo la tendencia a que por sí misma se erigiera una religión con sus dogmas, sus símbolos, sus altares y sus festividades. [...] Queda el hecho de que, en un caso determinado, se ha visto que la sociedad y sus ideas se convertían directamente, y sin transfiguración de ningún tipo, en objeto de un verdadero culto" (1982: 201).

Este proceso de sacralización transforma una realidad secular como la nación en algo sagrado. Ya hemos visto cómo originariamente "lo secular" fue parte de un discurso teológico (saeculum), donde las formas seculares se autonomizaron progresivamente de la esfera religiosa, pero, más tarde, se produce el proceso social inverso ya que, como consecuencia del proceso de secularización, la categoría de "lo religioso" emergerá de los discursos político-seculares y de los discursos científico-seculares, algo que se pone de manifiesto en estas nuevas sacralizaciones postaxiales modernas (T. Asad 2003: 192). Aunque "la función del secularismo como filosofía de la historia, y como ideología, (ha sido) convertir el proceso histórico particular cristiano occidental de secularización en un proceso teleológico universal de desarrollo humano que va de la creencia a la increencia, de la religión primitiva irracional o metafísica a la conciencia secular postmetafísica racional moderna" (J. Casanova 2012a: 213-214), sin embargo, no ha surgido un mundo postdualista que suponga hacer tabula rasa del dualismo secular-religioso, ya que "la tensión dinámica entre ambos polos sigue estructurando los procesos de trascendencia" que surgen en las sociedades modernas. Esta nueva metamorfosis de "lo religioso" sitúa a la religión como una categoría histórica y como un concepto universal globalizado dentro de los programas culturales y políticos de la modernidad secular occidental y de otras modernidades. 


\section{La Religión Civil americana}

Pero la efervescencia colectiva no es algo exclusivo de las convulsiones revolucionarias. El concepto de "religión civil americana" acuñado por Robert N. Bellah (2006: 225-245) ofrece un ejemplo genuinamente moderno de resacralización de la realidad secular de la nación. Mientras Durkheim nos ha ofrecido el irrenunciable ejemplo de la Revolución francesa, Bellah extraerá una serie de conclusiones asimismo irrenunciables a partir de "una colección de creencias, símbolos y rituales en relación a las cosas sagradas e institucionalizadas en una colectividad (la república americana)" (2006: 233). "Los hitos de emergencia de la "religión civil" se producen en medio de momentos de agitación social, de efervescencia colectiva, de crisis, que ponen a prueba la creatividad social de un colectivo, generando constelaciones de sentido instituyentes que la propia sociedad se encargará de institucionalizar". El primer estadio está representado por la guerra revolucionaria de independencia contra Inglaterra, donde George Washington emerge como el Moisés que conduce a su pueblo rompiendo las cadenas de la tiranía. El segundo estadio se forja en torno a la Guerra Civil, el "centro de la historia americana", momento que recoge la intensidad trágica de una lucha fratricida en una de las guerras más sangrientas del XIX. La Guerra Civil trajo consigo las cuestiones más profundas de significado nacional, y Abraham Lincoln, "nuestro presidente mártir" (ibidem, p. 236), aparecerá como el nuevo Jesús que recoge el testigo de Washington con nuevos desafíos a los que responder. El tercer estadio de desafío, Bellah lo sitúa en medio de la crisis por las consecuencias en la esfera pública de la Guerra de Vietnam y la efervescencia colectiva que genera el movimiento de los derechos civiles, con otro profeta emergente, Martin Luther King.

La religión civil es una comunidad nacional de culto pero no una comunidad de salvación, "es algo genuinamente americano y nuevo. Tiene sus propios profetas y sus propios mártires, sus propios acontecimientos sagrados y sus propios lugares sagrados, sus propios rituales solemnes y sus símbolos" (ibidem, 245), "el Dios de la religión civil no es un Dios "unitarista", ... (sino que) está más relacionado con el orden, la ley, la libertad y la justicia que con la salvación y el amor" ( $p$ : 232); de hecho, J. F. Kennedy, en su discurso inaugural, sitúa como enemigos, no a otros hombres sino, a la tiranía, la pobreza, la enfermedad y la guerra.

En este sentido, la religión civil es una religión postaxial, reordena la presencia de rasgos hebraicos y otros cristianos en un contexto nuevo. Bellah retoma el ejemplo de Tocqueville, en quien se inspira cuando afirma que la religión civil americana es una "religión democrática y republicana" (2006: 239)6. Según Tocqueville (1990: 309), el avance del racionalismo (es decir, la educación y el conocimiento científico) y del valor del individualismo (es decir, la democra- cia liberal y las libertades individuales) no conducen necesariamente a un declive de la religión. Es decir, Estados Unidos es un país secular y religioso a un tiempo; la Primera Enmienda de la Constitución no prohíbe a los trece Estados originarios que se practiquen en ellos "sus propias religiones establecidas". Lo que el Congreso veta es el establecimiento de una religión política oficial en los Estados Unidos. En la Norteamérica colonial no existió feudalismo ni una iglesia nacional extendida a lo largo de las trece colonias originarias de la que precisara separarse el nuevo Estado federal. Por tanto, la separación entre el Estado y la iglesia fue amistosa, no solo porque no había separación hostil en relación a una iglesia establecida preexistente, sino, porque la separación fue constituida para "proteger el libre ejercicio de la religión"; esto es, para construir las condiciones de posibilidad de un pluralismo religioso (denominacionalismo) (H. Richard Niebuhr 1929; 1957: 3-6, 1721, 25; J. Casanova 2012b) en donde se parte del supuesto de que la diversidad religiosa es un "bien" para la sociedad o la nación.

\section{La sacralidad emergente del proto-evento del 911}

El terrorismo no es solo una forma de acción política sino también una forma de acción "simbólica". El proto-evento del 11 de septiembre de 2001, en Nueva York, es un tipo particular de performatividad simbólica. Inicialmente, en el momento de la destrucción de las Torres Gemelas y del asesinato en masa de miles de inocentes, el acto terrorista, "ritualmente, significó" un atroz derramamiento de sangre -tanto literal como metafóricamente- haciendo uso de los fluidos vitales de las víctimas para arrojar una pintura beligerante y horrenda sobre el lienzo de la vida social (J. C. Alexander 2006: 91-115). En los términos de Austin podemos decir que el terrorismo es una fuerza ilocucionaria que pretende un efecto perlocucionario. No es la "cultura" en cuanto tal la que crea los guiones para la acción, sino los esfuerzos pragmáticos para proteger significados culturales particulares en pos de objetivos prácticos. A lo largo del tiempo histórico, y con consecuencias trágicas y terroríficas algunas veces, ha ido emergiendo gradualmente una tendencia pronunciada entre las civilizaciones judeocristiana e islámica a proyectar el mal en el otro, como algo exógeno a lo propio, creándose de esta guisa una distinción directriz que divide entre lo "sagrado/ amigo" propio y lo "profano/enemigo" ajeno, dentro de una lógica cultural de polarización exacerbada. En esta trama, Bin Laden asestó el primer golpe de forma carismática y "creativa", ya que controló inicialmente el despliegue de los medios de producción simbólica. Los actores-terroristas-mártires-suicidas tuvieron éxito destruyendo los iconos contaminados del capitalismo americano moderno, las Torres Gemelas, que evocativamente simbolizaron al enemigo occidental ateo. Pero, sin embargo, de esa pintura apocalíptica y dantesca, propia de Brueghel El Vie- 
jo, surge una comunión moral, "un contra-acto ritual transcultural, interracial e interclasista" -que agrupa a los bomberos, a los brókeres, a los policías, y coextensivamente a toda la sociedad americana y mundial detrás- en el que todo el mundo gritó, en medio de un gran rito expiatorio como signo de duelo: "'We are all americans now"', extendiéndose tanto la solidaridad nacional como la internacional. Ground Zero y 911 fungen como la contextura espacio-temporal icónica, es decir, el acto de destrucción de las torres y el holocausto de los inocentes les hace "convertirse de algo profano en algo sagrado", las cenizas de las torres y de los inmolados representan el "nuevo símbolo de fusión de la nación". Este ritual conmemorativo revive los puntos álgidos de intensidad emocional, los participantes se sienten implicados en ese centro de atención ritual (Randall Collins 2004: 53-87), simbolizado en los monumentos creados a tal efecto en el sur de Manhattan. Lo que es interesante subrayar, desde un punto de vista sociológico, es que aquello que ha creado un tipo de comunión moral, incluso en una sociedad funcionalmente diferenciada, no es el hecho de compartir todos las mismas creencias, puesto que Estados Unidos es una sociedad pluralista y diferenciada en lo cultural, sino el hecho de "compartir las mismas prácticas" en el seno de la esfera pública en la que una creatividad social emergente re-encantó y re-armó moralmente a la sociedad.

\section{LA SACRALIZACIÓN DE LA PERSONA HUMANA Y SU REFLEJo en los Derechos Humanos (1776- 1789 y 1948)}

La nación no es el único ámbito secular moderno que se ha sacralizado; la dignidad y el respeto a la persona humana se han convertido también en parte del núcleo sagrado de la sociedad moderna. Vamos a analizar los factores jurídicos, sociológicos y teológicos que intervienen en dicho proceso.

Podemos interpretar inicialmente la creencia en los derechos humanos y la dignidad humana universal como el resultado de un específico proceso de sacralización en el que todo ser humano ha sido convertido en algo sagrado, siendo institucionalizado dicho proceso en el "derecho", y generando crecientes y generalizados efectos de motivación y sensibilización dentro de la sociedad moderna. Si presuponemos esto, entonces, parece razonable entender los cambios en el sistema penal a partir de cambios en la comprensión de lo sagrado. Desde esta perspectiva, las reformas del derecho penal y la práctica penal, así como la creación de los derechos humanos a finales del siglo XVIII, son una expresión de un profundo cambio cultural a través del que "la persona humana se convierte en un objeto sagrado". Se le ha adscrito un nuevo significado a la sacralidad. La historia de los derechos humanos puede ser interpretada como una historia de sacralización (de la persona humana).
Aunque en el seno de las religiones universales -hinduismo, judaísmo, budismo, confucionismo, cristianismo e islamismo- está ya el germen de sacralidad de la vida humana, en la forma de un ethos del amor y del respeto universal al (prójimo) otro, el proceso de germinación de tal semilla se producirá en el seno de la Revolución Americana e inmediatamente después en la Revolución Francesa. Aunque la posición dominante ha considerado que los derechos humanos tienen su origen en un enfoque que procede de la llustración, según el cual el "carisma de la razón" (Weber 1978: 937) es la última forma histórica de carisma que procede de las fases originarias de la Revolución Francesa, y es la libertad individual el resultado que representa el mejor de los mundos; sin embargo, a juicio de Hans Joas (2011, cap. 1) ha sido Georg Jellinek en su obra intitulada La declaración de los derechos del hombre y del ciudadano: una contribución a la historia constitucional moderna, originariamente publicada en 1895, quien sitúa las raíces cristianas en la Declaración de los Derechos Humanos y no tanto a la llustración francesa, que ha sido escéptica, cuando no hostil, hacia la religión. Por tanto, en esta perspectiva, más que a una sacralización o carismatización de la razón, asistiríamos a una "sacralización o carismatización de la persona humana".

Las raíces intelectuales de los derechos humanos en el humanismo del Renacimiento, en la Reforma o en la Escolástica española son generalmente de menos ayuda en la comprensión del fenómeno que analizamos que la dinámica de su súbita institucionalización. Aquí es donde Jellinek observó la importancia decisiva que la libertad religiosa ha tenido para los protestantes americanos, especialmente para las congregaciones de inspiración calvinista, a los que Ernst Troeltsch (1931: 673) suma los grupos baptistas, cuáqueros y ciertas formas de espiritualidad libre. Así, Jellinek sitúa al héroe de su historia al pastor puritano Roger Williams, que abandona Massachusetts en 1632 para establecerse en Rhode Island, donde garantiza la libertad religiosa no solo de los cristianos de cualquier denominación sino también de judíos, paganos y turcos. La tesis central de Jellinek es que "la idea de los derechos inalienables, legalmente establecidos, sagrados e inherentes al individuo no tiene un origen político sino religioso. Lo que se ha mantenido como un trabajo de la Revolución Francesa fue en realidad un fruto de la Reforma y de sus luchas. Su primer apóstol no fue (el General) Lafayette sino Roger Williams, quien conducido por un profundo y poderoso entusiasmo religioso, se adentró en la naturaleza en orden a encontrar un gobierno de libertad religiosa y su nombre es proclamado por los americanos con el mayor de los respetos" (1979: 77. Énfasis mío). La idea de que los individuos no solo tienen derechos dentro de un Estado, sino también derechos contra el Estado, y de que estos derechos no son conferidos por el Estado, apunta a un origen 
religioso de los mismos. Esto aparece claramente en la Primera Enmienda de la Constitución de Estados Unidos, en donde Estado y religión están claramente diferenciados, pero en donde se protege y fomenta el libre ejercicio de toda creencia religiosa.

Un trabajo de Émile Durkheim de 1898, traído a colación por Hans Joas (2011, cap. 2), arroja mucha luz sobre el fenómeno que analizamos. Durante la agitación producida por el escándalo del caso Dreyfus en 1898, escribió: "Esta persona humana (personne humaine), cuya definición es como la piedra de toque que distingue el bien del mal, es considerada sagrada en el sentido ritual del mundo. Participa de la majestuosidad trascendente que las iglesias de todos los tiempos han atribuido a sus dioses; es concebida como un ser investido con tal propiedad misteriosa que crea un vacío en torno a las cosas sagradas, sacándolas del contacto vulgar y retirándolas de la circulación habitual. El respeto que se le da procede precisamente de esta fuente. Cualquiera que atenta contra la vida humana, contra la libertad humana, contra el honor humano, inspira en nosotros un sentimiento de horror análogo al que experimenta el creyente cuando observa que su ídolo ha sido profanado. Tal moral no es simplemente una disciplina higiénica o una buena economía de la existencia, es una religión donde el hombre es, a la vez, fiel y Dios"7 (Durkheim 1973: 46). Durkheim elaboró una teoría del cambio social y de sus fuerzas operantes pero no elaboró propiamente una teoría del "proceso" de sacralización de la persona humana. Él vincula las particularidades de la sociedad moderna a una energía creciente que procede de que "Ios sentimientos que tienen por objeto al hombre, se han vuelto muy fuertes [...] El grupo [...] es solo el medio para realizar y desarrollar la naturaleza humana [...] La calidad del hombre, [...], se ha convertido naturalmente en objeto por excelencia de la sensibilidad colectiva" (2006: 133). En otro pasaje Durkheim afirma: "Nada hay que el hombre no pueda amar y adorar en común sino a sí mismo. Así es como se ha convertido en un Dios para sí mismo y no puede ya crear otros dioses sin mentirse a sí mismo. $Y$ en la medida en que cada uno de nosotros encarna algo de la humanidad, cada conciencia individual contiene algo divino y se encuentra conformada por un carácter sagrado e inviolable ante otros" (1973: 52). Para Durkheim, "esta religión de la humanidad tiene todo lo que necesita para hablarle al creyente con un tono no menos imperativo que el usado por la religión que reemplaza [...] En realidad (el creyente) recibe su dignidad de una fuente más alta, de una que comparte con el resto de personas humanas. $\mathrm{Si}$ tiene derecho al respeto religioso, lo tiene gracias a que lo comparte con la humanidad"8 (ibidem, 48). La creación de los derechos humanos no es sino parte de este proceso de inclusión y de sacralización de la persona humana y de la coextensiva sacralización de la humanidad. Este concepto de sacralización de la persona no tiene nada que ver con la glorificación egocéntrica individualista liberal ( $\mathrm{y}$ utilitarista) del propio yo sino con la personalidad humana (Durkheim 1973: 45; H. Joas 2007: 151-168). Para Durkheim la "sacralidad de la persona" no es "un" sistema de creencias que contribuye a la integración social sino el "único" sistema de creencias que puede asegurar la unidad moral de un país.

La idea fuerza de esta nueva fe en la persona no es el egoísmo sino la simpatía por todo lo que el hombre representa, un gran sufrimiento por todos los dolores que aquejan al hombre, por las tragedias humanas, un compromiso para luchar contra ellas. La historia de la violencia y de la degradación humanas en 1776 en la Guerra de Independencia de Estados Unidos, en 1789 en la Revolución Francesa y en Europa en 1948, después de la espantosa Shoah, han conducido a adquirir una conciencia clara de que la dignidad de la persona es algo sagrado e inviolable. Pero, el sufrimiento, por sí mismo, no origina valores nuevos como el de la sacralización de la persona humana. Es preciso crear una "narrativa" $(H$. Joas 2011, cap. 3) que permita superar un acontecimiento horrendo, conectándolo con la creación de nuevos valores y significados que sean incorporados en el contexto social, religioso y político. De esta manera, un acontecimiento violento y traumático se convierte en un "trauma cultural" (J. C. Alexander 2012: 6-31) que recibe su base empírica a partir de la experiencia de los miembros de una colectividad que siente que su existencia ha sido amenazada en un acontecimiento horrendo que deja huellas imborrables en su conciencia de grupo y marca su memoria colectiva para siempre, cambiando su identidad futura de forma irrevocable. Las distintas narrativas de los derechos humanos, juegan, sin duda, este papel.

Esta idea no es extraña al propio cristianismo. El propio Durkheim lo pone de manifiesto en su trabajo de 1898: "El cristianismo expresa en una fe interior, en la convicción personal del individuo, la condición esencial de la divinidad...El centro de la vida moral ha sido así transferido de afuera hacia dentro $y$ el individuo se ha situado como el juez soberano de su propia conducta no precisando recurrir a otros criterios que no sean él mismo y su Dios"' (1973: 52). Es un error presentar esta sacralización de la persona humana y su correspondiente anclaje moral como antagonista de la moralidad cristiana. Todo lo contrario, aquella deriva de esta. Asumiendo aquella, no negamos nuestro pasado sino que lo continuamos. Durkheim seguía, con matices, una línea de argumentación que ya habían iniciado profetas de la Ilustración como Saint Simon y Comte cuando postularon que más tarde o más temprano una nueva religión de la humanidad (Durkheim 1973: 48) ${ }^{10}$ reemplazaría a las religiones teocéntricas.

Podemos ejemplificar esta idea en los desarrollos recientes experimentados por la propia iglesia católica 
a partir del Concilio Vaticano II (J. Casanova 2011). El proceso de transformación del catolicismo a lo largo de la segunda mitad del siglo $X X$, el proceso de su democratización interna, que se ha venido en llamar aggiornamento, confirma la visión de Durkheim sobre la sacralización de la persona operada en la modernidad avanzada, pero lo que ninguno de estos profetas y padres fundadores de la sociología positivista pudo haber anticipado es que, paradójicamente, los viejos dioses y las viejas religiones, cuya muerte Durkheim anunció ${ }^{11}$, han ganado nueva vida convirtiéndose en portadores del proceso de sacralización de la humanidad, como ocurre en el caso de la iglesia católica. "La sacralización de la persona y coextensivamente de la humanidad plasmados en la globalización de los derechos humanos ha emergido también en el seno de la propia iglesia católica a través de su propia secularización interna". Esto ha abierto el realineamiento de las relaciones entre los ámbitos religioso y secular.

Mientras las encíclicas anteriores fueron dirigidas en su mayor parte al creyente católico, comenzando con Pacem in Terris en 1963, los papas han tendido a dirigir sus pronunciamientos al mundo entero y a toda la gente. Desde un punto de vista teológico, "esto conlleva la transferencia del principio de la libertas ecclesiae (que le ha servido para utilizar su influencia ad hoc en conflictos entre Estados nacionales, asumiendo unas veces las perspectivas de estos y entrando en abierto conflicto con ellos en otros casos), que la iglesia ha guardado tan escrupulosamente a través del tiempo, a la persona humana individual, a la libertas personae" (Casanova 1997: 212 y ss.). En este proceso, el papa podría experimentar una curiosa transformación de ser el santo Padre de todos los católicos para convertirse en el padre común de todos los hijos de Dios y en el portavoz autoproclamado de la humanidad, en defensor hominis. El Papado ha estado intentando recrear el sistema universalista de la cristiandad medieval, pero ahora en una escala global. Quizás, la diferencia fundamental radica en que la espada del poder espiritual no puede ya buscar la protección de la espada del poder temporal con el objeto de ejercer su autoridad contra los regímenes religiosos en competencia, en orden a obtener el monopolio de los medios de salvación (lo cual dejaría sin valor el dogma católico del extra ecclesia nulla salus). ¿Qué sentido tiene hablar hoy de la iglesia como Una, Santa, Católica, Apostólica y Romana? El reconocimiento del principio de libertad religiosa significa que la iglesia ha aceptado competir en un sistema global relativamente abierto de regímenes religiosos. En este proceso de globalización, la iglesia católica-romana ha dejado de ser una institución predominantemente romana y europea, es decir, se ha deseuropeizado. Se ha producido un desplazamiento notable de la población católica del Viejo al Nuevo Mundo y del norte al sur. En los últimos pronunciamientos papales y episcopales, sobre todo en los relativos a asuntos de moralidad pública, el cambio fundamental es que no van dirigidos a los católicos como miembros fieles de la iglesia católica, obligados a seguir las normas específicas de la moral católica, "sino más bien a todos los individuos como miembros de la humanidad, obligados a seguir normas humanas universales, las cuales se derivan de los valores humanos universales de la vida y la libertad" (Casanova 1997: 220).

Sin duda, todo este conjunto de argumentos -jurídicos, sociológicos y teológicos- analizados proporcionan un sustento empírico al argumento lanzado por Talcott Parsons hace medio siglo en torno a la idea de una "generalización de valores" (1964: 29, 3, 339-357), y más en concreto a la generalización del valor sagrado de la persona humana.

\section{EL CHOQUE ENTRE LAS NARRATIVAS RESACRALIZADAS EN DISPUTA DE LA NACIÓN Y LA PERSONA HUMANA}

Las respectivas sacralizaciones de la nación y de la persona no forman parte de una mera pluralidad armónica de valores, de una pura coexistencia de constelaciones de valor modernas resacralizadas, como apunta P. L. Berger en su último libro (2014) citado al comienzo de este trabajo, sino que existe entre ellas una disputa interminable. La metáfora de la "lucha" (de los dioses) usada por Weber (y también por Simmel) ofrece un mejor acercamiento interpretativo a dos historias recientes que proceden de procesos sociales que han tenido lugar en Irlanda y en el País Vasco, en donde se ha producido un "choque entre estos dos tipos de narrativas modernas" que afectan a la nación y a la persona humana. Este párrafo de Weber refleja ese concepto de lucha simbólica: "El destino de una época que ha comido del árbol del conocimiento es que debe [...] reconocer que las nociones generales sobre la vida y el universo nunca pueden ser producto de un creciente conocimiento empírico, y que los más altos ideales que nos mueven con la mayor fuerza siempre se forman solo en la lucha con otros ideales que son tan sagrados para otros como lo son los nuestros para nosotros" (1982: 46).

Comencemos por desvelar la génesis histórico-social del choque de las dos sacralizaciones modernas de la nación y de la persona humana en la historia reciente de Irlanda. La publicación del Report of the Ryan Commission to Inquire into the Child Abuse ${ }^{12}$ en el 2009 detalló la existencia de numerosos casos de abuso sexual dentro del sistema irlandés de cuidado residencial de niños; otra fuente significativa de escándalo dentro del informe ha sido la parte relativa al olvido sistemático y a la crueldad a la que estaban sometidos los niños en las escuelas y reformatorios tutelados por la iglesia católica. La Comisión Ryan inició su andadura en 2000, siguiendo la estela de un programa de televisión, States of Fear, sobre la situación de los niños en residencias gestionadas por la iglesia católica, que tomaba a su vez el testigo de otro programa de radio, Dear Daughter, de 1996. 
Es a partir de 1921 cuando la iglesia católica se convierte virtualmente en el proveedor exclusivo de cuidado infantil en residencias del nuevo Estado irlandés, justo después del Alzamiento de Pascua de 1916. De esta manera, la formación de los niños se convierte en una práctica a través de la cual se materializa lo sagrado. A pesar de que las escuelas industriales fueron gestionadas por órdenes religiosas, sin embargo, fueron legalmente certificadas, inspeccionadas y subvencionadas por el Ministerio de Educación de Irlanda. A pesar de que las escuelas fueron establecidas para promover un entorno seguro de entrenamiento que sirviera a los niños como preparación para la vida adulta, sin embargo, los niños habitualmente las experimentaron como lugares de abuso y negligencia.

La fusión de catolicismo y nacionalismo, que adoptó una miríada de formas, configuró una "narrativa dominante" (Gordon Lynch 2012: 66-70; T. Inglis 2005: 64-65), que va desde las pretensiones que consideran a Irlanda como una civilización cristiana con una retórica política y religiosa (Brown 2010: 2627) hasta el uso del simbolismo católico con el objeto de celebrar el martirio de aquellos que han luchado por la libertad irlandesa (P. Murray 2000: 6-7; Conor C. O’Brian 1994). La República irlandesa, de esta guisa, sería el "santuario" donde surge el incienso sagrado de la devoción de Irlanda y de sus aspiraciones santas, el "camino" hacia ese destino que Dios tiene en mente para los niños de Gael (Murray, op. cit:: 411). La fortaleza y cohesión de la nación irlandesa descansa sobre su pureza moral y esta es inseparable de la piedad católica y sus costumbres sexuales. Cualquier sospecha sobre estas dos fuentes de legitimidad afectan a la plausibilidad de la narrativa dominante en torno a la nación irlandesa, por tanto, ese conjunto de "narrativas episódicas" creadas a contracorriente en el conjunto de programas de radio y televisión, así como los Informes Carrigan de 1931 y el más reciente de la Comisión Ryan, deben ser combatidas, a juicio de esta narrativa dominante. La forma sagrada de la nación católica irlandesa, que configura tanto las visiones imaginadas de la vida colectiva irlandesa como las subjetividades sexuales individuales, proporciona el recurso fundamental de legitimación simbólica dentro de los reformatorios para niños del sistema educativo irlandés. La nación pía debe separar las flores del mal, "lo moralmente impuro" (H. Ferguson 2007: 36, 132, 134), "lo moralmente contaminado" (ibid.: 130 y ss.).

Pero, como afirmamos en el apartado anterior, no basta con el sufrimiento para crear una nueva forma de ver las cosas, es necesario generar un conjunto de condiciones de posibilidad que permitan emerger una "nueva narrativa, un nuevo movimiento social y su incorporación en la cultura instituida" (G. Lynch 2012: 80 y ss.). Jeffrey Alexander ha desarrollado una noción de cultura propiamente entendida "no como una cosa sino como una dimensión, no como un objeto para ser estudiado como variable dependiente sino como una amenaza que corre a través de [...] toda forma social concebible" (2003: 7). La cultura sería más bien un conjunto de "construcciones fluctuantes" (ibid., 33), según las cuales las narrativas culturales no son algo estático sino "campos de fuerzas y contrafuerzas" implicados en luchas simbólicas.

En este sentido, una serie de factores sociológicos van a tener una gran importancia a la hora de configurar "el surgimiento de una vigorosa contranarrativa de la libertad inspirada en la sacralización de la persona humana representada en el niño", que encontrará su punto álgido en la década de los noventa, frente a la narrativa dominante del miedo representada por la sacralización de la nación católica irlandesa, que se ha proyectado a lo largo del siglo pasado. Entre estos factores podemos destacar, en primer lugar, la visita de Francis Edward Flanagan ${ }^{13}$ en junio de 1946, sacerdote norteamericano de origen irlandés, una celebridad tanto en Estados Unidos como en Irlanda por aquel entonces, que llegó al país para realizar un tour que incluyó intervenciones en la radio, presentaciones en las principales ciudades, encuentros con políticos. La discusión entre Flanagan y el establishment irlandés disparó el debate en la opinión pública, recogido en importantes contribuciones en el periódico Irish Times (ibid., 75). Segundo, la emergencia de la "niñez larga" (Ph. Ariés 1962: 329), esa fase de transición entre la infancia y la edad adulta, cuya existencia se despliega modernamente en Occidente, a partir del siglo XIX (antes solo existía la "niñez corta") con el desarrollo de nuevas instituciones educacionales y con el desarrollo del Estado de bienestar en el siglo XX, apuntala la singularidad de la niñez. Tercero, la emergencia de la deliberación democrática en la esfera pública en torno a temas relacionados con los discursos de la sexualidad contribuyó a eliminar temas monopolizados previamente por la hegemonía católica. Cuarto, la creación del primer canal de televisión en Irlanda permitió el desarrollo de espacios y prácticas mediáticos que acogen la crítica del poder institucional, luego continuada por Internet. Esto ha fortalecido la deliberación democrática. Quinto, los programas ya mencionados, States of Fear, Dear Daughter y sobre todo el impacto mediático e institucional del Informe Ryan, deslegitiman la posición dominante de la narrativa de la nación católica irlandesa y han creado una sensibilidad en torno a un tipo de sacralización centrada en la dignidad y el respeto hacia el niño.

En el caso del País Vasco, la disputa se objetiva entre la posición beligerante y profundamente antidemocrática representada por el terror ejercido en el nombre de la nación vasca por parte del nacionalismo radical a partir de la transición democrática española, que comienza su singladura en 1978 con la aprobación de la Constitución, y una narrativa que surge a finales de la década de los 90 y se agudiza a 
partir de 1997, apoyándose en la sacralización de la vida de ciudadanos inocentes sacrificados en el altar de la nación.

El primer nacionalismo vasco de finales del siglo XIX logra construir como problema social relevante en la esfera pública las distinciones directrices de "nosotros-ellos" y de "adentro-afuera" frente a la distinción directriz "arriba-abajo" que ya había producido el movimiento obrero. La atadura primordial sobre la que se estructura la idea que la sociedad tiene que tener sobre sí misma es Jaungoikoa (Dios celeste, Señor de Arriba), afirmándolo explícitamente: "Si en las montañas de Euskeria, antes morada de la libertad, hoy despojo del extranjero, ha resonado al fin en estos tiempos de esclavitud el grito de independencia, solo por Dios ha resonado" (S. Arana 1995: 168). Sabino Arana, padre fundador del primer nacionalismo vasco, construye un nivel de trascendencia en tres planos que se recoge en el acrónimo GETEJ (recogido en nota a sus "Apuntes íntimos"): $\mathrm{Gu}$ Euzkadirentzat ta Euzkadi Jaungoikoarentzat (Nosotros para Euzkadi y Euzkadi para Dios) (ibid., 20). El imaginario social religioso católico (Jaungoikoa) sirve de elemento simbólico de legitimación, en última instancia, para configurar una comunidad política imaginada (Euzkadi, el pueblo elegido) a su servicio, en la cual están integrados los miembros (los euzkos, los vascos).

Sin embargo, el nacionalismo vasco radical que surge a comienzos de los sesenta realizará un cambio: En lugar de Jaungoikoa (Dios) situará a Euskadi, o Euskalherria; el pueblo de la nación vasca se convertirá en esa realidad secular sacralizada a la cual quedarán referidas todas las ataduras primordiales que estaban anteriormente vinculadas al Dios monoteísta. George Steiner, en su obra Nostalgia del Absoluto, recurre a las expresiones: "credo sustitutorio" y "teología sustituta" para referirse a "sistemas de creencia y de razonamiento que pueden ser ferozmente antirreligiosos, que pueden postular un mundo sin Dios y negar la otra vida, pero, cuya estructura, aspiraciones y pretensiones respecto del creyente son profundamente religiosas en su estrategia y en sus efectos"14. ETA asumirá este planteamiento y lo hará suyo en el propio autonombrarse: Euskadi ta Askatasuna: Pueblo Vasco y Libertad, Jaungoikoa ha sido substituido por Euskadi -marcando una diferencia con el discurso de Arana-, un pueblo todavía sometido al yugo de la invasión, manteniendo esta semejanza con el discurso de Arana.

La violencia constituye el acta de su nacimiento y su exclusivo y permanente mecanismo de autoafirmación. "ETA no es una organización política que practica la violencia, sino un grupo armado que racionaliza políticamente sus acciones violentas" (J. Aranzadi 2001: 523). ETA define esta violencia ${ }^{15}$, autoadjudicada en régimen de monopolio frente al monopolio de la violencia ejercido por el Estado, como "una violencia pegajosa, demoledora, crónica, rentable, que nos haga cotizables", "no puede haber terror revolucionario sin una preparación escénica de tragedia, sin romanticismo de la muerte. El poder se toma por fascinación [...] solo la invocación y el hecho inminente de una gran tragedia colectiva es capaz de suscitar tal fascinación", "Euskadi se halla en estado de guerra contra España y Francia".

ETA adopta la violencia (desde su fundación, hasta el alto el fuego unilateral de 2011 , ha asesinado a 829 personas) no solo por razones de eficacia política sino también por su eficacia simbólica, por su "eficacia icónico-política"; pero, esta eficacia icónico-política se invierte de forma estrepitosa cuando asesina, de una forma deliberadamente despiadada, después de tenerlo secuestrado dos días intentando en vano chantajear a la opinión pública, al concejal del Partido Popular en Érmua, Vizcaya, Miguel Ángel Blanco de dos tiros en la cabeza, con las manos atadas a la espalda y lo abandona todavía agonizante en la cuneta de una carretera cerca de Lasarte.

Este asesinato representa un key event (Caminos, Armentia y Marín 2013: 140-160) ${ }^{16}$ un acontecimiento excepcional que desafía la posición dominante del discurso de la nación en el País Vasco para introducir una "narrativa alternativa" que procederá exitosamente a la resacralización de la persona, superando en eficacia icónica a los propios caídos de ETA. Este asesinato generará un "rito expiatorio de duelo nacional", en donde se hará patente un gran proceso de movilización colectiva, de efervescencia colectiva, donde se pondrá de manifiesto que ETA ha atentado "contra lo más sagrado", contra el corazón de la colectividad, la vida humana, generando un torrente de solidaridad que atraviesa las ideologías, las clases y los territorios. A partir de ese momento la legitimidad de la narrativa ritual violenta de la nación, apoyada en el terror, quedará herida de muerte. La muerte de Miguel Ángel Blanco se convirtió en un "protoacontecimiento apropiador" porque levantó a toda la sociedad vasca y española en defensa del valor sagrado de la vida humana, en buena parte, gracias a la resonancia mediática que supo transmitir a la esfera pública de manera inequívoca el valor añadido del impresionante "rito expiatorio de duelo" que convirtió la muerte profana de un inocente en un grandioso "símbolo sagrado" -en el que la colectividad con manos pintadas de blanco proclamó: "Nosotros somos Miguel Ángel Blanco"- contra el terror político comandado en el nombre de un grupo de autoproclamados héroes-asesinos nacionales. La muerte de Miguel Ángel Blanco no es otro asesinato más, es la "condensación ritual de un trauma cultural" (J. C. Alexander 2012) en el que comparecen la denuncia-rechazo, la energía emocional expresada, su dimensión estético-ritual y su grabación en la memoria colectiva (Birgitta Höijer 2004: 522). Este acontecimiento apropiador golpea la conciencia colectiva, 
la unifica y deja una marca indeleble en la memoria colectiva. El acto supuso la creación de una "imagen sagrada" encarnada en el cuerpo ultrajado de un inocente a través de un intercambio entre la acción ritual llevada a cabo por la gente y la representación de la narrativa visual de la muerte de Miguel Ángel Blanco recogida por todos los medios de comunicación de masas (Johanna Sumiala-Seppänen y Matteo Stochetti 2005: 228-249; Ron Eyerman 2011: 1-7). Cada año, ritualmente, como un ejercicio mnemónico insoslayable, la memoria colectica recuerda a través de actos públicos el valor sagrado de la vida humana copresente simbólicamente en la muerte cuasi martirial de Miguel Ángel Blanco.

\section{Notas}

1. Inspirándome, entre otras fuentes, en el modelo metodológico que introduce con el mismo nombre Hans Joas (2011, cap. 4).

2. Habitualmente se considera que el hecho religioso está basado en el uso de conceptos binarios (sagrado/ profano, trascendente/inmanente, religioso/secular) que son utilizados como conceptos sinónimos cuando en realidad no lo son. El par "sagrado/profano" representa categorías epistemológico-cognitivas, comunes a toda experiencia religiosa, tanto en el tiempo como en el espacio, que separan diferentes dominios del mundo. Lo sagrado es un ámbito interdicto, separado y extraordinario frente a lo profano. Émile Durkheim, Rudolf Otto y Mircea Eliade, entre otros, han explorado estas categorías. El par "este mundo"/"el otro mundo" representa categorías históricas forjadas en torno al siglo V a. C., dentro del surgimiento de la Edad Axial, cuyos portadores fundamentales son las "religiones universales" (World Religions). Cabe citar aquí, entre otras, las reflexiones de Max Weber, Karl Jaspers, S. N. Eisenstadt, Barry Schwartz y Robert Bellah. El par "religioso/secular" representa asimismo categorías históricas, pero esta vez nacidas dentro del cristianismo europeo occidental. En él coexisten, por una parte, el dualismo existente entre "este mundo" (la Ciudad del Hombre) y "el otro mundo" (la Ciudad de Dios) y, por otra parte, el dualismo existente dentro de "este mundo" entre una esfera "secular" y otra "religiosa". Ernst Troeltsch, P. L. Berger, David Martin, Charles Taylor, José Casanova y Talal Asad, entre otros, han analizado estas categorías.

3. É. Durkheim, -en adelante citaré abreviadamente FE por la cuarta edición francesa de PUF, 1960, y la edición española de Ramón Ramos, de Akal (1982)- FE: 308, 312-313/198, 205. Para una discusión interesante sobre la importancia del concepto "efervescencia colectiva" en la sociología durkheimiana y coextensivamente en el propio discurso sociológico, ver el trabajo de Pablo Nocera (2009): "Los usos del concepto de efervescencia y la dinámica de las representaciones colectivas en la sociología durkheimiana", pp. 93-119.9
4. É. Durkheim, FE: 312-13/205.

5. Albert Mathiez, en Los orígenes de los cultos revolucionarios (1789-1792), en un trabajo citado por Durkheim en Las Formas Elementales..., ofrece una interesante descripción del nuevo culto, de sus lugares y de sus tempos más relevantes.

6. La frase está tomada de Tocqueville (1990: 310).

7. Énfasis mío.

8. Énfasis mío.

9. Énfasis mío.

10. Ver A. Comte 1979, (1851-1854), Sistema... 97.

11. Ver É. Durkheim (1982: 398), Las formas elementales de la vida religiosa: "Los antiguos dioses envejecen o mueren, y todavía no han nacido otros (que los reemplacen)".

12. Report of the Ryan Commission to Inquire into the Child Abuse, 2006, vol. V, capítulo 5, 26-27, www.childabusecommission.ie/rpt/pdfs. (Acceso el 30 de abril de 2015).

13. Flanagan fue el fundador de la Ciudad de los Muchachos (Boys Town) y su trabajo recibió una gran atención pública gracias a la película de Hollywood, Boys Town, de 1938, por la que Spencer Tracy ganó un Óscar interpretando en su papel estelar al Padre Flanagan.

14. G. Steiner 2001: 16 y 19, citado en el libro de I. Sáez de la Fuente, El movimiento de liberación nacional vasco, una religión de sustitución, 2002, nota 8, pág. 62.

15. Estos fragmentos se encuentran en diversos Zutik de los años sesenta y en el folleto: "La insurrección en Euskadi". De todo ello encontramos extensa documentación en el trabajo de G. Jáuregui: Ideología y estrategia política de ETA, 1981.

16. Todos los medios de comunicación de masas, como muestra este trabajo, contribuyeron a crear una sólida corriente de opinión pública en defensa del valor de la dignidad de la persona y del respeto a la vida humana, proyectando la resonancia emergente de la intensificación de la vida colectiva que supuso el rito expiatorio de duelo. 


\section{Referencias Bibliográficas}

Alexander, Jeffrey. C. 2003. The Meanings of Social Life. A Cultural Sociology. Oxford: Oxford University Press. http://dx.doi. org/10.1093/acprof:oso/9780195160840.001.0001

Alexander, Jeffrey, C. 2006. "From the Depth of Despair: performance, counterperformance and 'September 11'". Pp. 91-115 en Social Performance. Symbolic action, Cultural Pragmatics and Ritual, editado por J. C. Alexander, Bernhard Giese y Jason Mast. Cambridge: Cambridge University Press.

Alexander, Jeffrey. C. 2012. Trauma. A Social Theory. Cambridge: Polity Press.

Arana, Sabino. 1995. La patria de los vascos. Donostia: Haramburu.

Aranzadi, Juan. 2001. El escudo de Arquíloco. Sobre Mesías, mártires y terroristas. Sangre Vasca. Madrid: Machado Libros.

Ariès, Philippe. 1962. Centuries of Chilhood. A Social History of Family Life. Nueva York: Vintage Books.

Asad, Talal. 2003. The Formations of the Secular. Christianity, Islam and Modernity. Stanford: Stanford University Press.

Bellah, R. N. y M. Tipton Steven. 2006. The Robert Bellah Reader. Durham: Duke University Press.

Berger P. L. 2014. The Many Altars of Modernity. Berlín: De Gruyter. http://dx.doi.org/10.1515/9781614516477

Brown, Theodor. 2010. Ireland. A Social and Cultural History, 1922-2001. Londres: Harper Perennial.

Caminos, J. M. ${ }^{\text {a }}$, J. I. Armentia y F. Marín. 2013. "El asesinato del Miguel Ángel Blanco como ejemplo de key event en el tratamiento mediático de los atentados mortales de ETA". Revista Estrategias 6:140-160.

Casanova, José. 1997. "Globalizing Catholicism and the Return to a 'Universal' Church". Pp. 121-143 en Transnational Religion and Fading States, editado por Suzanne Rudolph y James Piscatori. Boulder: Westview Press.

Casanova, José. 2011. "Cosmopolitanism, the Clash of Civilizations and the Multiple Modernities". Current Sociology 2:252-267. http://dx.doi.org/10.1177/0011392110391162

Casanova, José. 2012a. "Religion, the Axial Age and Secular Modernity". Pp. 191-221 en The Axial Age and its Consequences, editado por R. N. Bellah y Hans Joas. Cambridge Mass: Harvard University Press.

Casanova, José. 2012b. Genealogías de la secularización. Barcelona: Anthropos.

Collins, Randall. 2004. "Rituals of Solidarity and Security in the wake of Terrorist Attack". Sociological Theory 1:53-87. http://dx.doi.org/10.1111/j.1467-9558.2004.00204.x

Durkheim, Émile. [1912] 1960. Les formes elementaires de la vie religieuse. Le systéme totemique en Australie París: Librairie Félix Alcan.

Durkheim, Émile. 1973. "Individualism and the Intellectuals". Pp. 43-57 en Émile Durkheim on Morality and Society, editado por R. N. Bellah. Chicago: University of Chicago Press.

Durkheim, Émile. 2006. Lecciones de sociología. Física de las costumbres y del derecho. Granada: Editorial Comares.

Eyerman, Ron. 2011. The Cultural Sociology of Political Assassination. From MLK and RFK to Fortuyn and Van Gogh. Londres: Palgrave. http://dx.doi. org/10.1057/9780230337879

Ferguson H. 2007. "Abused and looked After Children as 'Moral Dirt': Child Abuse and Institutional Care in Historical Perspective". Journal of Social Policy 36:123-139. http://dx.doi.org/10.1017/S0047279406000407
Höijer, Birgitta. 2004. "The Discourse of Global Compassion: The Audience and the Media of Human Suffering". Media, Culture and Society 4:513-531. http://dx.doi.org/10.1177/0163443704044215

Hunt, Lynn. 1988. "The Sacred and the French Revolution". Pp. 25-44 in Durkheimian Sociology: Cultural Studies, edited by Jeffrey C. Alexander. Cambridge: Cambridge University Press. http://dx.doi.org/10.1017/CBO9780511598258.002

Inglis T. 2005. "Religion, Identity, State and Society". Pp. 5977 in The Cambridge Companion to Modern Irish Culture, edited by J. Crearly and C. Connolly. Cambridge: Cambridge University Press. http://dx.doi.org/10.1017/ CCOL052182009X.004

Jáuregui, Gurutz. 1981. Ideología y estrategia política de ETA. Madrid: Siglo XXI.

Jellinek, Georg. [1895] 1979. The Declaration of the Rights of man and of the Citizens: A Contribution to Modern Constitutional History. Wesport CT: Hyperion.

Joas, Hans. 2007. "Punishment and Respect: The Sacralization of the Person and its Endargement". Journal of Classical Sociology 2:159-177.

Joas, Hans. 2011. Die Sakralität der Person. Eine Neue Genealogie der Menschenrechte. Frankfort: Suhrkamp.

Llobera, J. R. 1994. The God of Modernity. The Development of Nationalism in Western Europe. London: Berg Publishers.

Lynch, Gordon. 2012. The Sacred in the Modern World: A Cultural Sociological Approach. Oxford: OUP. http://dx.doi. org/10.1093/acprof:oso/9780199557011.001.0001

Mathiez, Albert. [1904]. Les origins des cultes revolutionaires (1789-1792). Paris: Societé Nouvelle des Editions.

Murray, Peter. 2000. Oracles of God. The Roman Catholic Church and the Irish Politics, 1922, 1937. Dublin: UCD Press.

Niebuhr, Richard H. [1929] 1957. The Sources of Denominationalism. Nueva York: Living Age Books/Meridian.

Nocera, Pablo. 2009. "Los usos del concepto de efervescencia y la dinámica de las representaciones colectivas en la sociología durkheimiana". Revista Española de Investigaciones Sociológicas (REIS) 127:93-119.

O'Brian Connor, Cruise. 1994. Ancestral Voices. Religion and Nationalism in Ireland. Dublin: Poolberg Press.

Otto, Rudolph. 1985. Lo santo. Lo racional y lo irracional en la idea de Dios. Madrid: Alianza.

Parsons, Talcott. 1964. "Evolutionary Universals in Society". American Sociological Review 3:339-357. http://dx.doi.org/10.2307/2091479

Report of the Ryan Commission to Inquire into the Child Abuse. 2006, vol. V, capítulo 5, 26-27. Consultado el 30 de abril de 2015 (www.childabusecommission.ie/ $\mathrm{rpt} / \mathrm{pdfs}$ )

Sáez de la Fuente, Inmaculada. 2002. El movimiento de liberación nacional vasco, una religión de sustitución. Bilbao: Desclee Brower.

Schütz, Alfred. 1974. El problema de la realidad social. Buenos Aires: Amorrortu.

Schütz, Alfred y Thomas Luckmann. 1984. Strukturen der Lebenswelt. Francfort: Suhrkamp.

Simmel, Georg. 1986. "Puente y Puerta”. Pp. 29-35 en El individuo y la libertad. Barcelona: Península.

Simmel, Georg. [1918] 2000. "La trascendencia de la vida". Revista Española de Investigaciones Sociológicas (REIS) 89:297-315. 
Sieyes, Enmanuel J. [1789] 1970. Qu'est ce que le Tiers-Etat. Genève: Droz.

Steiner, George. 2001. Nostalgia del Absoluto. Madrid: Alianza.

Sumiala-Seppänen, Johanna y Matteo Stochetti. 2005. "Mediated Sacralization and the Postmodern Constitution of Communio Sanctorum: The Case of the Foreign Swedish Minister Anna Lindl". Material Religion 1:228-249. http://dx.doi.org/10.2752/174322005778054168

Taylor, Charles. 1998. "Modes of Secularism". Pp. 31-54 en Secularism and its Critics, editado por Rajeev Bhargava. Oxford: Oxford University Press.

Taylor, Charles. 2007. A Secular Age. Cambridge Mass: The Belknap of the Harvard University Press.

JOSETXO BERIAIN. Nacido en Idiazabal, Guipúzcoa, España, en 1959, de padres navarros. Es licenciado en sociología y en filosofía, máster en sociología por la New School for Social Research de Nueva York y doctor en sociología por la Universidad de Deusto. Actualmente es Catedrático de Sociología en la Universidad Pública de Navarra y Faculty Fellow del Center for Cultural Sociology de Yale University. Es autor, entre otros, de los siguientes libros: Representaciones colectivas y proyec-
Tiryakian, Edward. 1988. "From Durkheim to Managua: Revolutions as Religious Revivals". Pp. 44-66 en Durkheimian Sociology: Cultural Studies, editado por Jeffrey C. Alexander. Cambridge: Cambridge University Press.

Tocqueville, Alexis de. 1990. Democracy in America. Nueva York: Vintage.

Troeltsch, Ernst. 1931. The Social Teachings of the Christian Churches. Londres: Allen and Unwin.

Tweed Thomas, A. 2006. Crossing and Dwelling. A Theory of Religion. Cambridge Mass: Harvard University Press. http://dx.doi.org/10.4159/9780674044517

Weber, Max. 1978. Economía y sociedad. México D. F.: FCE.

Weber, Max. 1982. Ensayos de metodología sociológica. Buenos Aires: Amorrortu.

to de modernidad, Barcelona, Anthropos, 1990; La integración en las sociedades modernas, Barcelona, Anthropos, 1996; La lucha de los dioses en la modernidad, Anthropos, Barcelona, 2000; Modernidades en disputa, Anthropos Barcelona, 2005; Aceleración y tiranía del presente. La metamorfosis en las estructuras temporales de la modernidad, Barcelona, Anthropos, 2008, El sujeto transgresor (y transgredido). Modernidad, religión, utopía y terror, Barcelona, Anthropos-Siglo XXI, 2011. 